\title{
Microscopic cross sections: An utopia?
}

\author{
S. Hilaire ${ }^{1, a}$, A.J. Koning ${ }^{2}$, and S. Goriely ${ }^{3}$ \\ 1 CEA, DAM, DIF, 91297 Arpajon, France \\ 2 Nuclear Research and Consultancy Group, PO Box 25, 1755 ZG Petten, \\ The Netherlands \\ 3 Institut d'Astronomie et d'Astrophysique, Université Libre de Bruxelles, Campus de la Plaine, \\ CP 226, 1050 Brussels, Belgium
}

\begin{abstract}
The increasing need for cross sections far from the valley of stability poses a challenge for nuclear reaction models. So far, predictions of cross sections have relied on more or less phenomenological approaches, depending on parameters adjusted to available experimental data or deduced from systematical relations. While such predictions are expected to be reliable for nuclei not too far from the experimentally known regions, it is clearly preferable to use more fundamental approaches, based on sound physical bases, when dealing with very exotic nuclei. Thanks to the high computer power available today, all major ingredients required to model a nuclear reaction can now be (and have been) microscopically (or semi-microscopically) determined starting from the information provided by a nucleon-nucleon effective interaction. We have implemented all these microscopic ingredients in the TALYS nuclear reaction code, and we are now almost able to perform fully microscopic cross section calculations. The quality of these ingredients and the impact of using them instead of the usually adopted phenomenological parameters will be discussed.
\end{abstract}

\section{Introduction}

The TALYS code results from the collaboration between NRG Petten (the Netherlands) and CEA Bruyères-le-Châtel (France) initiated in 1998, with the objective to provide a complete and accurate simulation of nuclear reactions in the $1 \mathrm{keV}-200 \mathrm{MeV}$ energy range, through an optimal combination of reliable nuclear models, flexibility and user-friendliness. The development of TALYS has followed the following principle : "first completeness then quality". This does not mean that we use toy models to arrive at some quick and dirty results but rather that we have tried, in our quest for completeness, to divide our efforts equally among all nuclear reaction types. The precise description of all possible reaction channels in a single calculational scheme is indeed such an enormous task that we have chosen not to devote several years to the theoretical research and absolutely perfect implementation of one particular reaction channel which accounts for only a few millibarns of the total reaction cross section. Therefore, we aim to enhance the quality of TALYS equally over the whole reaction range and always search for the largest shortcoming that remains after the last improvement. TALYS-1.0 has been released at the conference on Nuclear Data for Science and Technology which was held in Nice in 2007 [1] and the current version, TALYS-1.2, has been recently distributed online. The first official release was the beta version TALYS-0.64 at the 2004 Nuclear Data conference held in Santa Fe [2]. During these six years, we could summarize the modifications/improvements that have occurred by changing the initial mantra into: "first completeness, now quality". In other words, TALYS-0.64,

\footnotetext{
a e-mail: stephane.hilaire@cea.fr
}

This is an Open Access article distributed under the terms of the Creative Commons Attribution-Noncommercial License 3.0, which permits unrestricted use, distribution, and reproduction in any noncommercial medium, provided the original work is properly cited. 
had somehow reached the searched completeness, and, since then, we have improved the quality of the models contained in the code, starting with better phenomenological default parameters for TALYS-1.0 to include more microscopic ingredients in the current TALYS-1.2 version.

\section{The microscopic ingredients}

One should not have the wrong feeling that TALYS-0.64 was not good enough to reproduce experimental cross section data. In fact, the completeness reached with this initial version provided one with basic default results that can be obtained specifying only the projectile, the target, and the incident projectile energy. This is due to an extensive database or/and to the use of systematics giving the unspecified parameters of the various models chained to produce the final cross section. These default models are generally based on analytical expressions depending on parameters which can be adjusted to fit experimental data. If, in practice, such fitting procedures enable one to fit experimental cross sections quite well, even for the complicated case of a fissile nucleus (see Fig. 1 in ref. [1]), the analytical expressions rely on approximations too simple to be able to represent the complexity of a nuclear reaction from a universal point of view. This is why we are now trying to provide alternatives to such analytical models by replacing them with microscopic or semi-microscopic ones. The final goal is to be able to reach roughly the same level of accuracy as with the phenomenological approaches when experimental data are available together with a greater confidence in the quality of the predictions when no data are available. We are indeed convinced that (semi)-microscopic approaches have higher predictive power than extrapolations of phenomenological formulae adjusted on a very narrow range of nuclei in the valley of stability. Before discussing the impact of using these (semi)-microscopic ingredients on cross section predictions, let's first briefly recall what and how good these ingredients are.

\subsection{Ground state properties}

The starting point of any predictions in a unknown region of the nuclear chart is a nuclear structure model, as fundamental as possible, which provides the basic properties of a nucleus, starting with its binding energy. To this end, several iterations have been performed for years to improve the description of nuclear masses - using the self consistent Hartree-Fock-Bogoliubov method with an effective nucleon-nucleon interaction of the Skyrme type - incorporating step by steps other constraints such as level densities, fission barriers etc ... The price to pay was sometimes a slight deterioration of the mass fit which was however compensated by a gain on other properties. The mass model which has been included in TALYS, is based on the BSk14 Skyrme interaction [3]. This model is used to obtain the nuclear masses, the ground state deformation, spin, parity, as well as other information out of which nuclear model ingredients can be constructed, such as level densities, fission barriers, optical potentials or $\gamma$-strength function. As far as the nuclear binding energies are concerned, the rms deviation is not the best one obtained within the framework of such self consistent approaches, but the fission barriers are much better described than in previous studies (see Ref. $[3,4]$ and references therein).

\subsection{Nuclear level densities}

Another good illustration of the idea underlying our quest for quality is shown by the nuclear level densities. All traditional analytical expressions assume at least (i) simple approximations to describe shell, pairing and deformation effects, (ii) that the spin distribution is given by the derivative of a Gaussian distribution and (iii) that there is an equipartition of positive and negative parity levels. These last two approximations are clearly wrong for low energies and it has been shown $[5,6]$ that departures from these two approximations could have a direct impact on the cross section predictions. Therefore, we have added the combinatorial level densities in TALYS, first as determined in Ref. [6] and then as in Ref. [7] to suppress some approximations made in Ref. [6]. These microscopic level 
densities are tabulated as a function of the excitation energy, spins and parities, and provide results of similar quality to those obtained using global analytical expressions [7]. However, one has to keep in mind that a code like TALYS is not only of academic interest, but is also used to fit cross sections, in particular by adjusting level densities. This is the reason why we have given a recipe to also play with our tabulated values to (i) improve the description of experimental level density data if necessary and/or (ii) eventually fit experimental cross sections. This recipe has been recently used [8] to provide systematics of adjustable factors used to improve simultaneously the description of the low energy discrete levels as well as the experimental mean s-wave neutron resonance spacings.

\subsection{Optical model potentials}

Another ingredient which governs the prediction of nuclear cross sections is the optical model potential (OMP). Again, the problem is similar to the level density problem. When experimental data are available, a phenomenological OMP reproduces much better the observables than a microscopic or semi-microscopic OMP. Following this idea, a global OMP has been developed [9] and selected as the default one in TALYS-0.64. An improvement of TALYS-1.0 was the inclusion of the global deformed OMP of Ref. [10] which is used as the default for actinides. With this scheme, coupledchannels calculations for various types of deformation (symmetric-rotational, harmonic-vibrational, vibration-rotational, and asymmetric-rotational) can be automatically performed. However, this does not guaranty that extrapolations of these phenomenological OMPs far from the valley of stability are reliable. An alternative to this weakness is to use the semi-microscopic OMP of Ref. [11] which has proven its predictive power. This semi-microscopic OMP is based on the knowledge of nuclear matter densities which are calculated within the Hartree-Fock-Bogolyubov (HFB) approach and tabulated in the TALYS structure database. As for the level densities, we believe that OMPs derived from sound physical bases, which in turn can compete with highly parameterized phenomenological models to reproduce experimental data globally, are certainly more reliable when going far from the regions where measurements have been performed. In practice, the semi-microscopic model included in TALYS reproduces experimental data within roughly $5 \%$ whereas a phenomenological model with fine-tuned parameters can reach less than $1 \%$ of accuracy.

\section{$2.4 \gamma$-ray strength functions}

The gamma ray strength function can also be obtained out of the same nucleon-nucleon effective interaction following the method described in [12]. Again, several models depending on adjustable parameters had been included in TALYS-0.64, and now, the new option based on tabulated microscopic HFB plus QRPA predictions is available. As shown in [13], such microscopic gamma ray strength may provide better results when compared to experimental data than the default phenomenological models. On top of that, as can be seen in Fig. 1, if, qualitatively speaking, the microscopic strength functions rather look like the phenomenological Lorentzian for nuclei close the valley of stability, this is not at all the case for exotic neutron rich nuclei. Such differences may of course have very large impact on cross section predictions.

\subsection{Fission barriers}

Last but not least are the fission barriers which can also be microscopically determined coherently with all the previously mentioned ingredients. As pointed out in Ref. [3], the barriers determined with the HFB-14 model reproduce the 52 primary barriers of nuclei with $88 \leq Z \leq 96$ (which are always less than $9 \mathrm{MeV}$ high), with an rms deviation as low as $0.67 \mathrm{MeV}$. A similar accuracy is obtained $(0.65 \mathrm{MeV})$ for the secondary barriers. Generally speaking, as for the other nuclear ingredients, powerful phenomenological approaches, representing the fission barriers by multiple-humped inverted parabolic shapes (the height and curvature of which, among other parameters, are adjusted to fit fission cross sections) were implemented in TALYS-0.64. Again, if this picture is qualitatively in agreement 


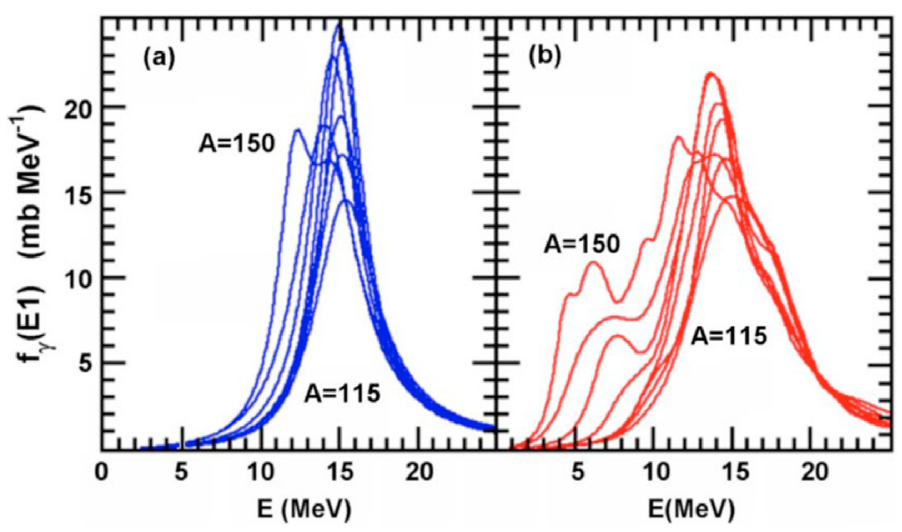

Fig. 1. Comparison between (a) the phenomenological model [14] implemented in TALYS and (b) the microscopic E1- $\gamma$ strength functions of Ref. [12] for Sn isotopes.

with microscopic predictions, as shown in Ref. [15], the fission path for exotic neutron-rich nuclei cannot, in general, be simply approximated by such simple shapes. To estimate the transmission coefficients with fission barriers deviating from the simple inverted parabolic picture, the full WKB method $[16,17]$ has been implemented in the updated version of TALYS to deal with arbitrary tabulated fission barrier shapes, as those deduced from the HFB-14 model.

\section{Impact of the microscopic ingredients on cross sections}

All the above-mentioned microscopic nuclear ingredients are included in TALYS-1.2 and provide alternatives to the traditional phenomenological expressions used to predict nuclear reactions. The only ingredient left is the particle-hole level density for which we still use analytical expressions and for which the inclusion of a microscopic model is presently in progress. Otherwise, we are now able to predict cross sections that depend only on a nucleon-nucleon interaction (this is made possible through the use of tables for all these ingredients). The advantage of using microscopic ingredients instead of analytical ones has been discussed several times both for nuclear level densities $[5,18]$ and $\gamma$-ray strength functions [13]. One important impact of combinatorial level densities, for instance, is the fact that neither the parity equipartition nor a Gaussian spin distribution are assumed. This last property can have a significant impact in particular when considering nuclear reactions populating isomeric levels [5].

In Fig. 2, we plot a fine-tuned calculation of cross section using usual analytical level densities (full lines) and just replace the analytical level densities by the microscopic ones (dotted lines). This comparison is the illustration of the predictive power of microscopic models, since it compares on the one hand a calculation where all nuclear ingredients have been tuned to fit the cross sections, and on the other hand a blind calculation for the microscopic level densities. Yet, the impact is not very important, which shows that the raw level densities are close to an optimum, at least for the (n,xn) channels. Indeed, when looking at the (n, $\gamma$ ) channel known to be very sensitive to the nuclear level density, the situation is quite different. In particular, as shown in Fig. 3(b), the calculated capture cross section using the raw microscopic level densities (black line) does not describe the experimental data very well, as does the analytical level density (blue line). As can be seen in Fig. 3(a), the raw microscopic level density do not describe very well the experimental discrete levels either. If the recipe of Ref. [8] is employed to improve the agreement between the microscopic level densities and the known low energy discrete levels (red line), it turns out that the experimental capture cross sections are also better reproduced, almost as well as with the fine-tuned calculation. There are certainly cases where the microscopic inputs will provide worse results than in the present case, but we believe that, in general, the quality of the microscopic ingredients is reasonably good, and, certainly better than the phenomenological extrapolations when going far from the experimentally accessible regions. 
EFNUDAT Workshop ”Measurements and Models of Nuclear Reactions", Paris, 2010

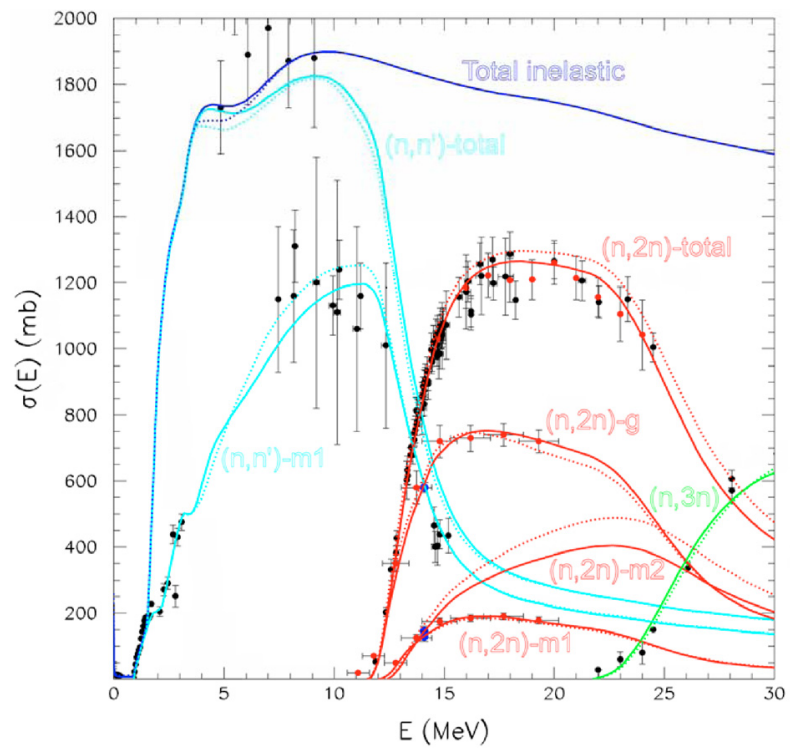

Fig. 2. Cross sections obtained with a fine-tuned analytical level density (full lines) and with the combinatorial model (dotted line) for several channels in the $\mathrm{n}+{ }^{89} \mathrm{Y}$ reaction.
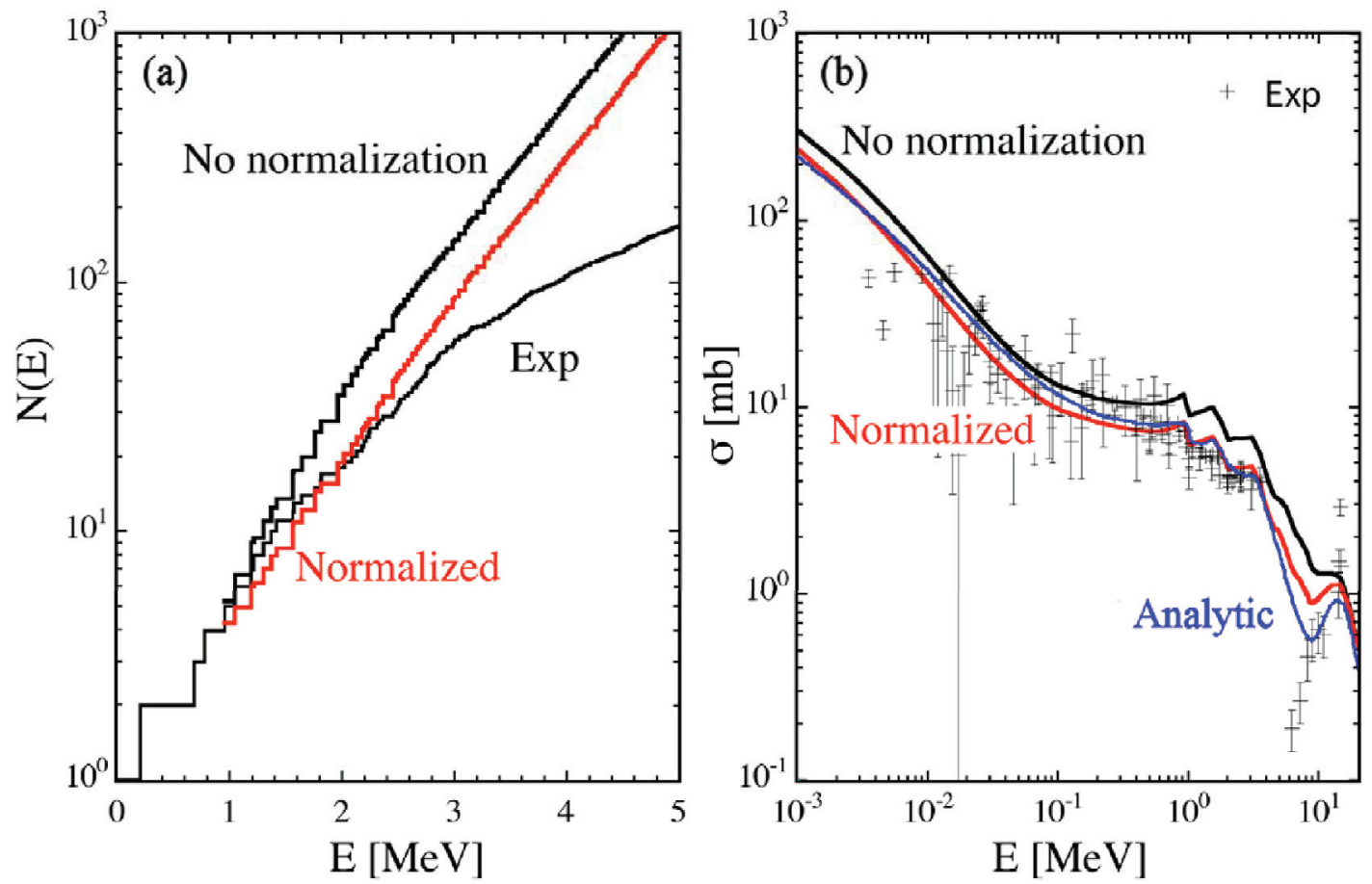

Fig. 3. Correlation between the level density of ${ }^{90} \mathrm{Y}$ and the neutron capture cross section for ${ }^{89} \mathrm{Y}$ (see text for details).

Last but not least is the fission cross section prediction. If very accurate fits of fission cross section can be achieved [16,19], it is mainly thanks to the use of a very large number of parameters which are generally not strongly constrained by experimental data but rather adjusted to reach a good accuracy. 
More than in any other channel, the predictive power of the traditional approaches is poor, and by no mean such approaches can be employed to extrapolate far from the regions where fission has been measured. The only solution left in this case is to rely on microscopic predictions provided they give reasonable answers. Quite a complete study has been performed on the use of microscopic ingredients applied to fission cross section prediction [20] and concludes that the quality obtained by default is not satisfactory for practical applications which require a few percent of accuracy. This is mainly due to the fact that the microscopic barriers are generally too high by a few hundreds of keV $[3,20]$ which is too big an error to provide reasonable cross sections. Yet, if the barriers are individually normalized, it is then possible to obtain cross sections which are in much better agreement with experimental data. However, such normalizations only make sense if experimental data are available. If not, it is still possible to use systematic normalizations deduced by averaging those which have been obtained fitting the nuclei for which experimental data are available. In that case, one obtains fission cross section which are globally within a factor of 3 versus experiment.

\section{Conclusions}

The TALYS code has reached the completeness initially expected and we are now improving the quality of the database on which the code relies. Thanks to the high computer power available nowadays, systematic microscopic calculation have been performed not only for fundamental research but also for practical applications. We had anticipated the eventual arrival of new microscopic models when we started to develop TALYS, and we are now incorporating them in order to see how accurate they are in cross section calculation. The final goal is to be able to make a real link between the fundamental effective nucleon-nucleon interaction and the cross section calculation. The only ingredients which are not yet fully determined microscopically are the partial level densities required in the preequilibrium model. This improvement is under progress. For all the other nuclear ingredients, we have included several tables in the database which can be used as alternatives to the traditional analytical ingredients.

Another important remaining issue is the semi-microscopic optical model potential. Right now, it is only automated for spherical nuclei. A future improvement which we plan is to include the treatment for deformed nuclei within this framework.

Last but not least is the question of the effective nucleon-nucleon interaction. Indeed the currently implemented tables rely on a Skyrme force which suffer from limitations. An improvement would consist in using a finite range interaction with good pairing properties such as the Gogny interaction. Work in this direction has already be initiated by improving the quality of the Gogny force predictions with respect to nuclear masses [21] and also by investigating the level density predictions obtained using this new Gogny interaction. The results are quite promising and we are thus planning to include, one by one, the ingredients required to perform microscopic cross section calculation out of an optimized Gogny interaction. We believe that work along such a path is a way to progress and be able, in the future, to obtain satisfactory evaluations on the basis of reliable and accurate microscopic inputs only.

\section{References}

1. A.J. Koning, S. Hilaire, and M. Duijvestijn, Nucl. Data for Science and Technology (EDP Sciences, eds Bersillon et al.), 211 (2008); see also http://www.talys.eu.

2. A.J. Koning, S. Hilaire, and M. Duijvestijn, Nuc. Data for Science and Technology (Amer. Inst. of Physics, New York, USA, eds R. Haight et al.), 1154 (2005).

3. S. Goriely, M. Samyn and J.M. Pearson, Phys. Rev. C, 75, 064312 (2007).

4. S. Goriely et al., These proceedings.

5. S. Goko et al., Phys. Rev. Lett., 96, 192501 (2006).

6. S. Hilaire and S. Goriely, Nucl. Phys. A, 779, 63 (2006).

7. S. Goriely, S. Hilaire and A.J. Koning, Phys. Rev. C, 78, 064307 (2008).

8. A.J. Koning, S. Hilaire and S. Goriely, Nucl. Phys. A, 810, 13 (2008). 
9. A.J. Koning and J.P. Delaroche, Nucl. Phys. A, 713, 231 (2003).

10. E. Sh. Soukhovitskii et al., J. Phys. G, 30, 905 (2004).

11. E. Bauge, J.P. Delaroche and M. Girod, Phys. Rev. C, 63, 024607 (2001).

12. S. Goriely, E. Khan, and M. Samyn, Nuc. Phys. A, 739, 331 (2004).

13. H. Utsunomiya et al., Phys. Rev. Lett., 100, 162502 (2008).

14. J. Kopecky and M. Uhl, Phys. Rev. C, 41, 1941 (1990).

15. S. Goriely and J.M. Pearson, Proc. of the Inter. Conf on Nuclear Data for Science and Technology (EDP Sciences, eds O. Bersillon et al.), 203 (2008).

16. M. Sin et al., Phys. Rev. C, 74, 014608 (2006).

17. S. Goriely et al., Phys. Rev. C, 79, 024612 (2009).

18. M. Segawa et al., Phys. Rev. C, 76, 022802 (2007).

19. M.J. Lopéz Jiménez et al., Ann. Nucl. Energy, 32 (2005) 195.

20. S. Goriely et al., Phys. Rev. C, 79, 024612 (2009).

21. S. Goriely, S. Hilaire and M. Girod, Phys. Rev. Lett., 102, 242501 (2009). 